\title{
Viabilidade Técnica e Financeira de implantação de um sistema fotovoltáico em uma Instituição de Ensino Superior particular em Cajazeiras - PB
}

\author{
Technical and Financial Feasibility of implementing a photovoltaic system in a private Higher \\ Education Institution in Cajazeiras - PB \\ Viabilidad Técnica y Financiera de la implementación de un sistema fotovoltaico en una Institución \\ de Educación Superior privada en Cajazeiras - PB
}

Recebido: 30/04/2021 | Revisado: 06/05/2021 | Aceito: 11/05/2021 | Publicado: 27/05/2021

Michael Douglas Sousa Leite ORCID: https://orcid.org/0000-0002-9356-1872

Faculdade Católica Santa Teresinha, Brasil E-mail: michaeldouglas_adm@hotmail.com

Kaio Luís de Azevêdo Santos ORCID: https://orcid.org/0000-0002-5904-8549

Faculdade Católica Santa Teresinha, Brasil E-mail: kaioluis@hotmail.com

Sandra Maijane Soares de Belchior ORCID: https://orcid.org/0000-0001-5807-2259

Faculdades Integradas do Ceará, Brasil

E-mail: sandrabelchior@hotmail.com

Kadydja Mayara Ramos Nobre ORCID: https://orcid.org/0000-0003-2269-1220

Universidade Federal de Campina Grande, Brasil E-mail: kadydja_mrn@hotmail.com

Pablo Sthefano Roque de Souza Bandeira ORCID: https://orcid.org/0000-0002-8980-5977

Universidade Regional do Cariri, Brasil E-mail: bandeiraroque@gmail.com

Aline Cristina de Araújo Florentino Silva ORCID: https://orcid.org/0000-0002-0091-6946 Faculdade de Filosofia, Ciências e Letras de Cajazeiras, Brasil E-mail: alineflorentino.fasp@gmail.com

Gisely Gabriela Bezerra de Sousa ORCID: https://orcid.org/0000-0002-0010-0541

Faculdades Integradas do Ceará, Brasi

E-mail: giselysousa@sousaadvocacia.com

Júlia Marcia Lourenço de Almeida Martins Medeiros ORCID: https://orcid.org/0000-0001-8554-8136 Universidade Federal de Campina Grande, Brasil E-mail: juliaalmeidamartins@gmail.com

Messias Rodrigues Ramos ORCID: https://orcid.org/0000-0003-0209-9414 Universidade Federal de Campina Grande, Brasil E-mail: messias.rodrigues@tecnico.ufcg.edu.br Verônica Cristian Soares de Belchior ORCID: https://orcid.org/0000-0003-2357-9599 Faculdades Integradas do Ceará, Brasil

E-mail: prof_veronicabelchior@hotmail.com

Wendell de Almeida Lacerda ORCID: https://orcid.org/0000-0002-9737-1013 Faculdades Integradas do Ceará, Brasil E-mail: wendelllacerada@gmail.com

Alberto Grangeiro de Albuquerque Neto ORCID: https://orcid.org/0000-0002-1632-1808 Instituto Federal de Educação, Ciência e Tecnologia da Paraíba, Brasil

E-mail: albertograngeironeto@hotmail.com Jose Ramon Nunes Ferreira ORCID: https://orcid.org/0000-0002-8764-2641 Universidade Federal de Campina Grande, Brasil E-mail: joseramonnunes@gmail.com 


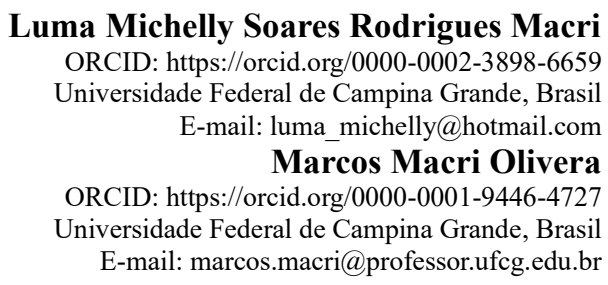

\begin{abstract}
Resumo
Em um contexto em que o desenvolvimento consciente se mostra cada dia mais necessário, a rentabilidade financeira de projetos com eficiência tecnológica e ambiental ganha destaque, e é vista como fator determinante para o crescimento. Pensando nisso, o aproveitamento da energia originada pelo sol, tanto enquanto fonte de calor como de luz, é propicio para fornecer toda a energia em termos de eletricidade. Assim sendo, essa pesquisa tem como objetivo desenvolver um estudo de viabilidade técnica econômico-financeira de implantação de um sistema fotovoltaico em uma Unidade de Ensino Superior Particular em Cajazeiras - PB. Para tanto a presente pesquisa trata-se de um estudo de caso, exploratório e descritivo de abordagem quantitativa. Os resultados mostraram que o prazo para o retorno de investimento está em 3,8 anos e que se espera que haja uma economia de cerca de $\mathrm{R} \$ 2.753 .000,00$ nos próximos 25 anos, o que torna a instalação do sistema bastante atrativa. Sendo assim, é evidente o benefício que a utilização das fontes alternativas pode trazer tanto para as organizações, quanto ao meio ambiente, beneficiando a população como um todo. O Brasil tem um enorme potencial quanto o assunto, que é energia solar e com certeza pode ser mais bem explorada por todos segmentos da indústria/comércio brasileiro e mundial.
\end{abstract}

Palavras-chave: Análise financeira; Investimento; Geração fotovoltaica; Recursos Naturais; Viabilidade.

\begin{abstract}
In a context in which conscious development proves to be more and more necessary, the financial profitability of projects with technological and environmental efficiency is highlighted and is seen as a determining factor for growth. With that in mind, the use of energy originated by the sun, both as a source of heat and light, is conducive to supply all the energy in terms of electricity. Therefore, this research aims to develop a technical economic and financial feasibility study for the implementation of a photovoltaic system in a Private Higher Education Unit in Cajazeiras - PB. For this purpose, this research is a case study, exploratory and descriptive with a quantitative approach. The results showed that the term for the return on investment is 3.8 years and that it is expected that there will be savings of approximately $\mathrm{R} \$$ $2,753,000.00$ over the next 25 years, which makes the installation of the system quite attractive. Thus, it is evident the benefit that the use of alternative sources can bring both to organizations and to the environment, benefiting the population as a whole. Brazil has enormous potential on the subject, which is solar energy and it can certainly be better explored by all segments of Brazilian / global industry / commerce.
\end{abstract}

Keywords: Financial analysis; Investment; Photovoltaic generation; Natural resources; Viability.

\title{
Resumen
}

En un contexto en el que el desarrollo consciente resulta cada vez más necesario, se destaca la rentabilidad financiera de proyectos con eficiencia tecnológica y ambiental y se vislumbra como un factor determinante para el crecimiento. Teniendo esto en cuenta, el uso de la energía originada por el sol, tanto como fuente de calor como de luz, es propicia para suministrar toda la energía en términos de electricidad. Por tanto, esta investigación tiene como objetivo desarrollar un estudio de viabilidad técnica y económica para la implementación de un sistema fotovoltaico en una Unidad Privada de Educación Superior en Cajazeiras - PB. Para ello, esta investigación es un estudio de caso, exploratorio y descriptivo con enfoque cuantitativo. Los resultados mostraron que el período de retorno de la inversión es de 3,8 años y que se espera un ahorro de aproximadamente R \$2.753.000,00 en los próximos 25 años, lo que hace bastante atractiva la instalación del sistema. Así, es evidente el beneficio que el uso de fuentes alternativas puede traer tanto a las organizaciones como al medio ambiente, beneficiando a la población en su conjunto. Brasil tiene un enorme potencial en el tema, que es la energía solar y ciertamente puede ser mejor explorado por todos los segmentos de la industria / comercio brasileño / global.

Palabras clave: Analisis financiero; Inversión; Generación fotovoltaica; Recursos naturales; Viabilidad.

\section{Introdução}

A procura por fontes de energia que sejam renováveis é um dos desafios enfrentados pela sociedade nos últimos anos e vem ganhando importância com a preocupação com o meio ambiente. Uma das formas promissoras de contornar essa problemática é aproveitar a energia fornecida pelo sol, fonte limpa e gratuita de energia (Dantas \& Pompermayer, 2018).

Siqueira (2015) relata que o aproveitamento da energia originada pelo sol, tanto enquanto fonte de calor como de luz, é propicio para fornecer toda a energia em termos de eletricidade. No Brasil a fonte principal de eletricidade é ainda de usinas 
hidrelétricas. No entanto, em períodos de escassez prolongados de chuva, há um risco de racionamento de eletricidade, o que está relacionado aos reservatórios das barragens das usinas atuais. Uma das soluções que vem sendo adotada a este desafio é a diversificação da sua matriz energética, em particular com geradoras eólicas e usinas termoelétricas.

Em razão do desenvolvimento tecnológico e da redução dos custos dos painéis solares, este tipo de tecnologia está difundido amplamente em diversos países como: China, Estados Unidos, Alemanha, Espanha, entre outros. No Brasil, houve evolução principalmente após a resolução nº 482, de 17 de 2012, da ANEEL, que apresentou melhores condições para as fontes incentivas de energia. E ainda, com à crise hídrica atual, o governo vem adotando medidas para estimular a instalação de micro e minigeradores solares fotovoltaicos (Brasil, 2012).

De acordo com Alves \& Lira (2018) a Energia Solar vem crescendo bastante, no entanto, no Brasil ainda é pouco explorado, tendo muito espaço ainda para evoluir. Com sol praticamente o ano inteiro, o território brasileiro é um campo vasto a ser explorado. De acordo com ENEEL (2020) no primeiro trimestre de 2019, foram conectados e instalados à rede de energia elétrica mais de 14,000 sistemas, sendo que ao final do corrente ano, ultrapassou a marca de 74.000 sistemas instalados e conectados, mostrando assim a média de crescimento fotovoltaico no Brasil. A estimativa da Empresa de Pesquisa e Energia (EPE), é que em 2027 o Brasil estará com 1,4 milhão de consumidores.

Colaborando Dantas \& Pompermayer (2018) relatam que a energia solar para as pequenas empresas está sendo visualizada como um meio por abrir possibilidades novas de investimentos sustentáveis, para a diminuição de custos e maior sustentabilidade. Os autores destacam que hoje as empresas tem uma grande dificuldade em pagar a energia convencional, pelo fato da mesma ser altíssima, pois é a quinta energia elétrica mais cara do mundo. Com isso atrapalha para que as empresas possam investir mais em algumas produções até para estoque, investir em máquinas, devido ao consumo ser alto e muita das vezes não consegue vender seu produto fabricado pelo preço que deveria ser, pelo fato de se tornar caro, demissões no quadro de funcionários e etc. A empresa que investe num projeto de Energia Solar, ela tende a pagar até menos em um financiamento comparado ao que paga a concessionária de energia, levando em consideração que o financiamento é por pouco tempo, levando assim a possibilidade de as empresas poderem investir mais.

Desta forma, esse trabalho se desenvolveu a partir do seguinte questionamento de pesquisa: Existe viabilidade técnica e financeira de implantação de um sistema fotovoltaico em uma Unidade de ensino Superior particular em Cajazeiras-PB?

Assim sendo, essa pesquisa tem como objetivo desenvolver um estudo de viabilidade técnica econômico-financeira de implantação de um sistema fotovoltaico em uma Unidade de Ensino Superior Particular em Cajazeiras - PB. Para a concretização e entendimento do objetivo geral, foram traçados objetivos específicos, como tópicos importantes para a formulação do objetivo geral, sendo estes: Analisar o consumo energético em relação à capacidade de geração de energia por meio de sistemas de energia fotovoltaicos; Dimensionar o sistema fotovoltaico; Prever os procedimentos de instalação do sistema e, assim, a viabilidade técnica; Analisar a viabilidade econômica através de parâmetros como o Payback, o Valor Presente Líquido (VPL) e a Taxa Interna de Retorno (TIR).

O presente estudo é justificado pelo contexto em que o desenvolvimento consciente se mostra cada dia mais necessário, a rentabilidade financeira de projetos com eficiência tecnológica e ambiental ganha destaque e é vista como fator determinante para o crescimento. Nesse sentido, estudos de viabilidade são primordiais para a continuidade do crescimento do setor com uma visão alternativa e racional (Cabral \& Vieira, 2012; Schossler, 2018).

$\mathrm{O}$ aproveitamento dos recursos naturais apresenta um desenvolvimento surpreendente dentro de diversos setores nos últimos anos, tendo em vista que a adesão de uma gestão com responsabilidade socioambiental tem se mostrado diretamente proporcional à rentabilidade de uma empresa. Dessa forma, a presente pesquisa justifica-se pela atual necessidade em estudar o retorno financeiro dos sistemas de aproveitamento da energia solar fotovoltaica, aliando-o à rentabilidade ambiental a curto e longo prazo e, assim, contribuindo com o crescimento da exploração dessa fonte de energia ampla, limpa e inesgotável 
(Schossler, 2018).

A abundante disponibilidade de recursos e incentivos governamentais e a fácil utilização deles para conversão de energia por meio de células fotovoltaicas são agravantes positivos para esse desenvolvimento e a viabilidade financeira tem, a cada dia mais, atraído a atenção de empresas, visando altos lucros em um setor com constantes avanços tecnológicos e ainda em ascensão (Maia, 2018). Com a hipossuficiência dos consumidores em relação à iniciativa privada, é perceptível a falta de conhecimento desses em relação à viabilidade dos sistemas que the são comercialmente disponibilizados (Schossler, 2018).

Porém, ainda se espera maior adesão à essa tecnologia em países como o Brasil, devido ao seu grande potencial de radiação solar, com médias anuais que variam entre $4 \mathrm{kWh} / \mathrm{m} 2$ e $6,5 \mathrm{kWh} / \mathrm{m} 2$ por dia, enquanto em países como Alemanha o valor máximo é de 3,44kWh/m2 (Alves, et al., 2014). Vários fatores contribuem para essa resistência, como o alto custo para implantação do sistema, que demanda grande investimento inicial; e políticas públicas e tarifárias, em que o incentivo ainda deixa a desejar. Dessa forma, estudos da viabilidade de implantação de energia solar têm sido fundamentais para a sua consolidação na matriz energética brasileira e mundial. Estes têm trazido contribuições importantes para uma maior eficiência e consequente redução de custos de sistemas fotovoltaicos, bem como a sua popularização ao ressaltar seus benefícios à economia e ao meio ambiente a curto e longo prazo (Mori, 2018).

Assim, ao executar o estudo proposto em uma unidade de ensino, acredita-se incentivar o uso da tecnologia, ampliando o conhecimento da mesma e contribuindo para uma possível implantação no futuro. Esta que é uma instituição com alta visibilidade, onde transitam milhares de pessoas; além de contar com um consumo significativo de energia elétrica durante o dia e noite.

\section{Metodologia}

A pesquisa em análise trata-se de um estudo de caso, exploratório e descritivo de abordagem quantitativa. Segundo Marconi \& Lakatos (2012) utiliza-se estudo de caso com o propósito de buscar informações e conhecimentos a respeito de um problema, para o qual se busca uma resposta, ou de uma hipótese, que se queira comprovar, ou descobrir novos fenômenos ou as relações entre eles.

O estudo descritivo segundo Malhotra (2014) é descrever algo, principalmente as características e funções de determinado item. De acordo com Oliveira (2012) a pesquisa quantitativa indica a quantificação de dados, empregando dados estatísticos, numerando e avaliando dados.

O cenário estudado localiza-se em uma Unidade de Ensino Superior Particular na Cidade de Cajazeiras, município que ocupa uma área total de $586,275 \mathrm{~km}^{2}$ e possui uma população de 58.319 habitantes. Encontra-se situada na extremidade ocidental do estado da Paraíba, no Alto Sertão. Limita-se a norte com São João do Rio do Peixe, oeste com cachoeira dos Índios e Bom Jesus, ao sul com são José de Piranhas, a noroeste de Santa Helena e sudeste de Nazarezinho (IBGE, 2012).

A instituição estudada foi instituída em 19 de janeiro de 1965 e veio atender à necessidade de se implantar uma Faculdade no Sertão da Paraíba, objetivando dirimir a carência de profissionais qualificados para o ensino de $1^{\circ}$ e $2^{\circ}$ graus, e ainda dar oportunidade aos jovens sertanejos que não tinham como frequentar um curso superior na Capital do Estado.

Os dados foram coletados através de pesquisa documental das faturas de energia elétrica, pesquisa de campo, pesquisa bibliográfica a partir de livros, periódico, normativos e resoluções visando identificar a contextualização do campo de estudo e fundamentar os aspectos tecnológicos relacionados ao desenvolvimento do projeto de energia solar fotovoltaica e consequentemente o estudo dos indicadores de viabilidade econômica.

Para o dimensionamento da usina fotovoltaica verificou-se a viabilidade técnica da instalação como: rede elétrica compatível com a geração de energia, visibilidade direta para o Sol, estrutura do telhado para suportar as placas fotovoltaicas e espaço para instalar as mesmas. 
Após estas análises, verificou-se o consumo médio em KWh durante os 12 meses de 2018 (janeiro a dezembro). Para análise quantitativa utilizou-se Matlab para realizar os cálculos da geração de energia, usando as seguintes informações: localização no qual a instalação se encontra; irradiação solar no local (horas de sol equivalente/dia), obtido através do programa SUNDATA; número total de painéis solares, de acordo com a área total do telhado disponível para instalação dos equipamentos; e potência e tensão elétrica dos painéis solares.

Por fim, utilizou-se o Microsoft Excel (2016) para realizar a análise econômico-financeira do sistema proposto, identificando qual será o retorno do investimento ao longo da vida útil das placas. Os dados obtidos depois da compilação dos dados foram expostos em gráficos e tabelas para a melhor compreensão e discutidos segundo a literatura pertinente.

\section{Resultados e Discussão}

Para se fazer o dimensionamento do sistema fotovoltaico é preciso conhecer a demanda em questão de energia do local. Assim, foram analisados através do histórico de consumo de energia elétrica computados pela concessionária local a ENERGISA.

Foram analisadas as faturas de energia ao longo de 12 meses (janeiro a dezembro de 2018). Escolheu esse período por representar o ano letivo, bem como em função da pandemia do COVID-19 que desde do dia 18/03/2020 a instituição está funcionando em grande parte remotamente, o que reduz o consumo de energia. Dessa forma, foi possível detectar a diferença no consumo de energia de acordo com a época do ano, ou seja, durante as férias espera-se um menor consumo de energia devido à menor circulação de pessoas; e durante o verão também se esperam um maior consumo devido à utilização de ventiladores e ar-condicionado, por exemplo.

Um dos fatores de grande influência na geração de energia elétrica de um sistema fotovoltaico é o posicionamento do painel e a ocorrência de sombreamentos. Dessa forma, para se obter a maior eficiência possível do sistema, é necessário analisar bem o local ideal para a instalação dos módulos. A princípio, os telhados parecem ser mais indicados, porém se faz necessário analisar a inclinação e orientação a fim de se escolher o mais indicado (Marchioro, Dahmer \& Souza, 2018).

No caso estudado os paneis foram instalados nos telhados visto ser uma área grande e com boa inclinação para o sol como mostra a figura $01 \log 0$ abaixo.

Figura 1 - Foto via satélite do teto da Instituição depois da aplicação dos painéis

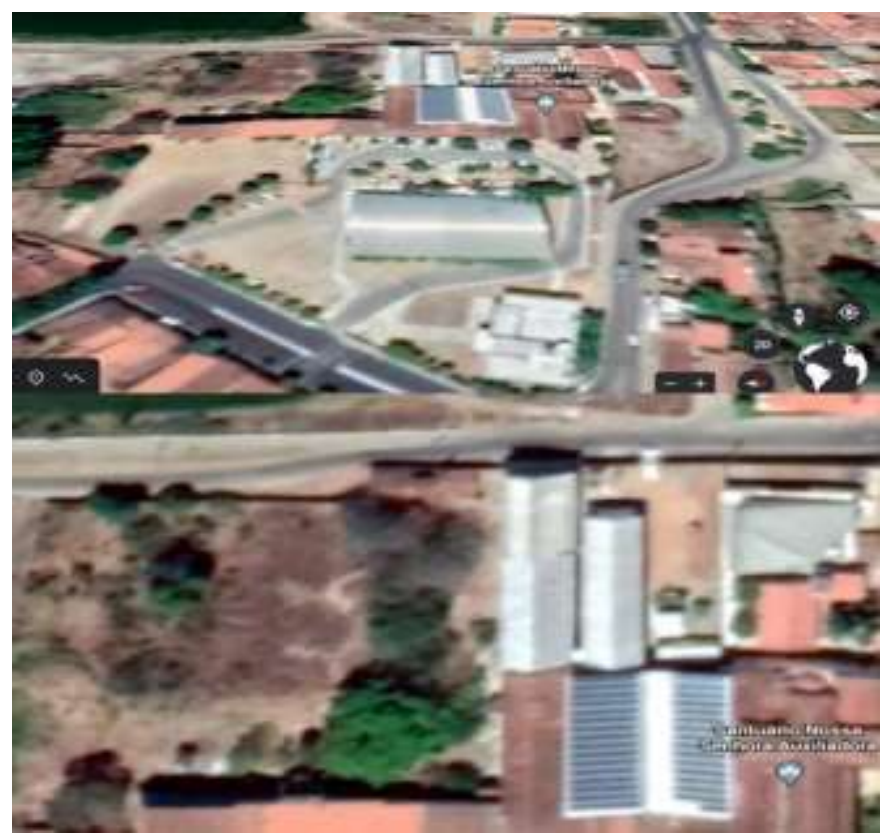

Fonte: Google Earth (2020). 
Vale destacar que foi necessário considerar as estruturas de suporte das placas no orçamento. Foi usado a solução integrativa, onde sistema passa a ser parte da edificação (Sonai et al., 2015).

O sistema a ser instalado à rede não possui engrenagens, partes móveis ou qualquer tipo de motor. Sendo assim, a manutenção está restrita à verificação de conectores e equipamentos, e a realização de limpezas, principalmente quando houver acúmulo excessivo de resíduos ou até mesmo de poeira. A água da chuva já se encarrega de uma limpeza recorrente (Marchioro, Dahmer \& Souza, 2018).

A Figura 2 mostra de uma maneira geral os principais equipamentos utilizados para a instalação do sistema fotovoltaico. Com a quantidade de equipamentos utilizados, estima-se que a área ocupada para instalação foi de aproximadamente $2500 \mathrm{~m}^{2}$.

Basicamente, o sistema é formado por painéis fotovoltaicos, inversor grid TIE e fixadores das placas para o telhado. Inversores Grid Tie são inversores de corrente utilizados pelo sistema. Os inversores são capazes de fazer uma sincronização entre o sistema e a rede pública de eletricidade, garantindo que a energia gerada seja fornecida exatamente como aquela que usualmente recebemos da rede elétrica convencional.

Figura 2 - Foto via satélite do teto da Instituição depois da aplicação dos painéis.

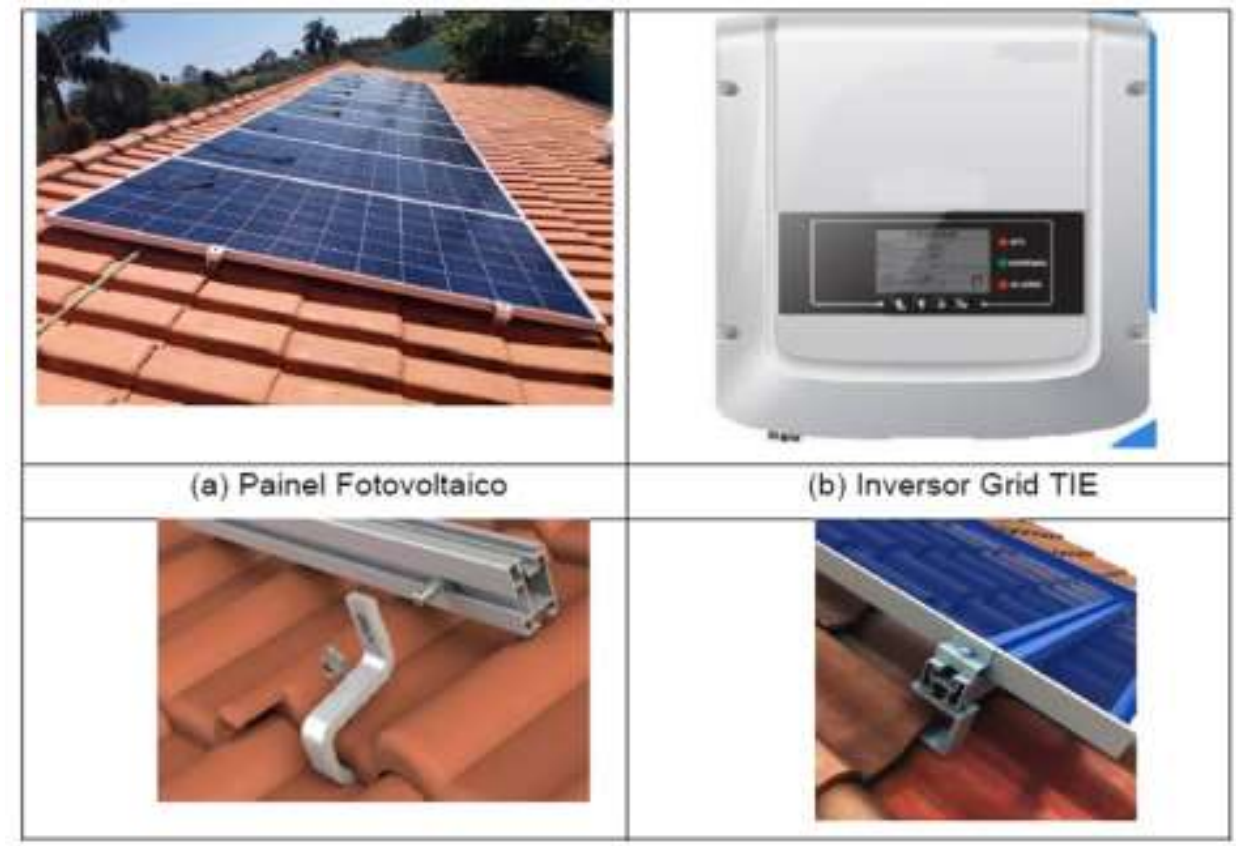

Fonte: Cruz (2020).

O inversor tem a função de transformar a energia solar captada pelos painéis em energia elétrica convencional para uso no dia a dia. Ele também é capaz de garantir a não ocorrência de conflitos entre essas duas fontes de energia. No entanto é preciso que haja uma boa sincronização e as devidas proteções elétricas.

Há um monitoramento da energia via WEB. O sistema de monitoramento WEB permite ao usuário acessar remotamente o sistema e verificar o que está sendo gerado. Além de monitorar por meio da WEB, o usuário também tem a opção de acesso via smartphone.

A irradiação solar do local de estudo é mais uma característica importante para se conhecer para o melhor dimensionamento do sistema de geração solar fotovoltaica. O Brasil apresenta médias anuais relativamente altas em todo o seu território, a cidade de Cajazeiras - PB, está com média anual de 5,808 kWh/m2.dia.

A referida cidade está situada nas coordenadas geográficas: latitude -6,890153, longitude -38,544097 e altitude 295 . Na Tabela 1 são apresentados dados climáticos das médias mensais. 
Tabela 1 - Dados climatológicos para Cajazeiras - PB.

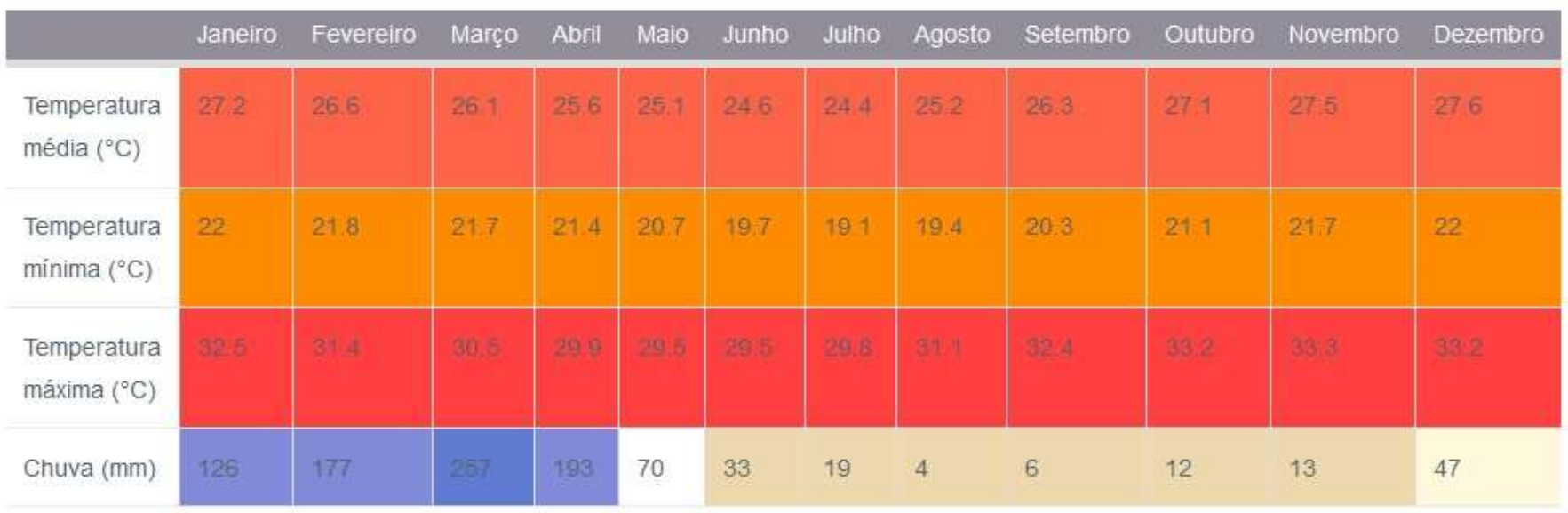

Fonte: Climate Data.Org (2020).

Analisando a tabela é possível perceber que dezembro é o mês mais quente do ano com uma temperatura média de 27.6 ${ }^{\circ} \mathrm{C}$. Ao longo do ano Julho tem uma temperatura média de $24.4^{\circ} \mathrm{C}$ sendo durante o ano é a temperatura média mais baixa.

O gráfico 1 mostra abaixo a média de consumo em KW da instituição pesquisada ao longo de 2018.

Gráfico 1 - Variação do Consumo em KW no ano de 2018.

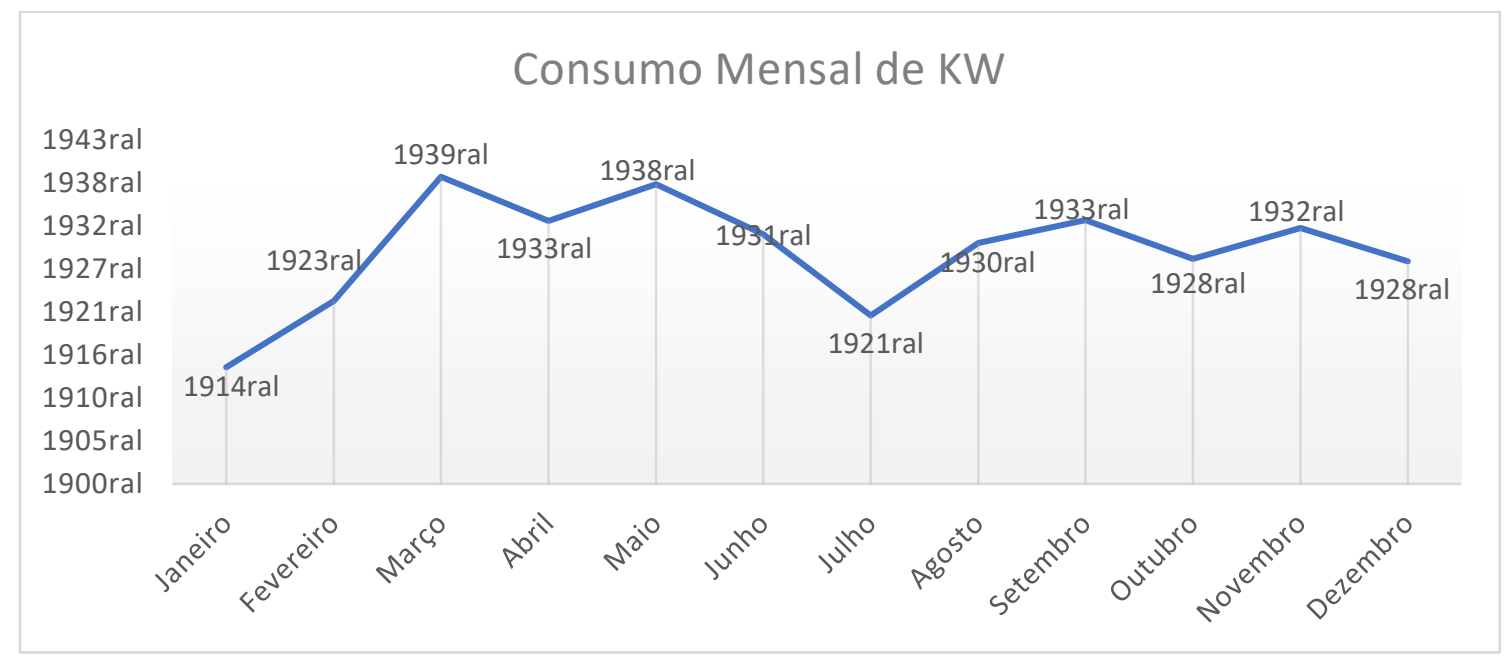

Fonte: Dados da Pesquisa (2020).

Como mostra no gráfico acima o mês de maior consumo foi o mês de março com $14.305 \mathrm{~kW}$ e o meses com menor consumo foram janeiro $5.433 \mathrm{~kW}$ e junho $7.849 \mathrm{~kW}$ pois é quando os alunos estão de férias.

Ainda analisando a viabilidade técnica como forma comparativa, a Tabela 2 mostra várias características para uma escolha correta dos módulos. Os dados importantes a serem observados, além do próprio custo (no nosso caso, custo relativo), são baixo coeficiente de temperatura e eficiência das células (que implicará na menor área do módulo em função da potência gerada). 
Tabela 2 - Opções de inversores no mercado.

\begin{tabular}{llllll}
\hline Marca & Modelo & Valor (R\$) & Potência Máxima (W) & $\begin{array}{l}\text { Tensão Máxima } \\
(\mathrm{V})\end{array}$ & $\begin{array}{l}\text { Garantia } \\
(\text { anos })\end{array}$ \\
\hline Growatt & & & 450 & 10 \\
Fronius Galvo & $1500-\mathrm{S}$ & 2152,74 & 1500 & 420 & 10 \\
Ecolys & $1.5-1$ & 4570,02 & 1600 & 340 & 10 \\
\hline
\end{tabular}

Fonte: Dados da Pesquisa (2020).

O inversor selecionado instalado foi o modelo 1500-S da marca Growatt, pois como pode ser visto na Tabela 3 ele apresenta a potência máxima superior a encontrada como sendo a máxima de pico do string, a tensão máxima de entrada é superior a tensão de circuito aberto do string e seu valor foi o menor dentre os três que foram apresentados.

Abaixo destaca-se o custo do projeto, vale ressaltar que esses valores são baseados no preço de mercado.

Tabela 3 - Custo do projeto implementado.

\begin{tabular}{ll}
\hline Equipamentos & Valor total (R\$) \\
\hline Painéis & $329.453,00$ \\
Inversor & $27.830,00$ \\
Demais equipamentos & $42.717,00$ \\
Mão de obra e outros custos & $15.000,00$ \\
\hline Custo total & $\mathbf{4 1 5 . 0 0 0 , 0 0}$ \\
\hline
\end{tabular}

Fonte: Dados da Pesquisa (2020).

Para os custos relativos ao projeto, os valores de aquisição são os considerados os para pagamento à vista. Outra opção que será mostrada logo mais são as diversas linhas de financiamento de energia solar que são oferecidas, hoje, através de bancos públicos e privados, muitas das quais permitem pagar a parcela com a própria economia obtida na conta de luz, tornando a aquisição dos sistemas fotovoltaicos mais acessível para boa parte da população.

Apesar de todos os evidentes benefícios da implantação da energia solar fotovoltaica, o fator econômico é determinante para a viabilidade. Assim, como explicado nos objetivos, foram utilizados três parâmetros: VPL (Valor Presente Líquido), TIR (Taxa Interna de Retorno) e Payback.

Para que tal análise seja possível é preciso considerar: o custo de implantação do sistema de geração de energia elétrica por painéis fotovoltaicos; os custos incorridos com a perda de eficiência dos painéis fotovoltaicos até o período de análise temporal do projeto; o valor mínimo pago a concessionária de energia elétrica para que a instituição pesquisada se mantenha integrada ao sistema público; a taxa mínima de atratividade para o capital investido na implantação do sistema fotovoltaico e o custo anual de energia elétrica da instituição (Assaf Neto, 2010).

Nesta análise não é considerado o crescimento da demanda ao longo dos anos futuros, pois implicaria na necessidade de considerar também o aumento do sistema de produção de energia fotovoltaica. Tal consideração não é inviável, pois a projeção de demanda, considerando o crescimento da estrutura do campus poderia ser realizada considerando o planejamento estratégico da Instituição.

No entanto, a queda no preço dos equipamentos fotovoltaicos e o aumento de sua eficiência, características que vem evoluindo de forma sistemática, acabariam por inserir um viés não desejável nesta análise, por ser esta evolução de difícil mensuração em função da seguida onda de novas tecnologias que vem sendo desenvolvidas e adotadas. 
Para efeito desta análise, considerando os custos com os próprios painéis e com a instalação estimou-se o valor médio de mercado $\mathrm{R} \$ 415.000,00$, a partir do contato com fornecedores. Assim, sendo uma geração de 15.000kWh/mês, necessária para atender a demanda de pico da Instituição.

Um sistema de produção de energia fotovoltaica não pode prescindir de procedimentos de manutenção preventiva e corretiva, pois são equipamentos que estão sob ação de agentes externos como poeira, radiação solar, movimentação mecânica por força dos ventos e própria degradação de conectores, cabos, equipamentos inversores, que exigem algum tipo de atuação.

Quanto aos custos incorridos pela perda de eficiência na produção de energia fotovoltaica, autores como Krauss et al., (2015) e Ishii \& Massuda (2017) descrevem como mais relevantes dois agentes denominados Degradação Induzida pela Luz, o que traz uma perda de cerca de $1 \%$ ao ano.

Abaixo destaca-se o cálculo simples da economia ao longo dos 25 anos, vale destacar que nesse primeiro exemplo não são considerados taxa de juros e inflação.

Tabela 4 - Cálculo Simples da Economia ao longo dos 25 anos.

\begin{tabular}{|c|c|c|c|c|}
\hline Ano & $\begin{array}{c}\text { Valor Gasto em } \\
\text { Energia/Ano } \\
\end{array}$ & Rendimentos dos painéis & Valor Gerado & Economia ao longo dos anos \\
\hline 1 & $\mathrm{R} \$ 144.000,00$ & $100 \%$ & $\mathrm{R} \$ 144.000,00$ & $-\mathrm{R} \$ 271.000,00$ \\
\hline 2 & $\mathrm{R} \$ 144.000,00$ & $99 \%$ & $\mathrm{R} \$ 142.560,00$ & $-\mathrm{R} \$ 128.440,00$ \\
\hline 3 & $\mathrm{R} \$ 144.000,00$ & $98 \%$ & $\mathrm{R} \$ 141.120,00$ & $\mathrm{R} \$ 12.680,00$ \\
\hline 4 & $\mathrm{R} \$ 144.000,00$ & $97 \%$ & $\mathrm{R} \$ 139.680,00$ & $\mathrm{R} \$ 152.360,00$ \\
\hline 5 & $\mathrm{R} \$ 144.000,00$ & $96 \%$ & $\mathrm{R} \$ 138.240,00$ & $\mathrm{R} \$ 290.600,00$ \\
\hline 6 & $\mathrm{R} \$ 144.000,00$ & $95 \%$ & $\mathrm{R} \$ 136.800,00$ & $\mathrm{R} \$ 427.400,00$ \\
\hline 7 & $\mathrm{R} \$ 144.000,00$ & $94 \%$ & $\mathrm{R} \$ 135.360,00$ & $\mathrm{R} \$ 562.760,00$ \\
\hline 8 & $\mathrm{R} \$ 144.000,00$ & $93 \%$ & $\mathrm{R} \$ 133.920,00$ & $\mathrm{R} \$ 696.680,00$ \\
\hline 9 & $\mathrm{R} \$ 144.000,00$ & $92 \%$ & $\mathrm{R} \$ 132.480,00$ & $\mathrm{R} \$ 829.160,00$ \\
\hline 10 & $\mathrm{R} \$ 144.000,00$ & $91 \%$ & $\mathrm{R} \$ 131.040,00$ & $\mathrm{R} \$ 960.200,00$ \\
\hline 11 & $\mathrm{R} \$ 144.000,00$ & $90 \%$ & $\mathrm{R} \$ 129.600,00$ & $\mathrm{R} \$ 1.089 .800,00$ \\
\hline 12 & $\mathrm{R} \$ 144.000,00$ & $89 \%$ & $\mathrm{R} \$ 128.160,00$ & $\mathrm{R} \$ 1.217 .960,00$ \\
\hline 13 & $\mathrm{R} \$ 144.000,00$ & $88 \%$ & $\mathrm{R} \$ 126.720,00$ & $\mathrm{R} \$ 1.344 .680,00$ \\
\hline 14 & $\mathrm{R} \$ 144.000,00$ & $87 \%$ & $\mathrm{R} \$ 125.280,00$ & $\mathrm{R} \$ 1.469 .960,00$ \\
\hline 15 & $\mathrm{R} \$ 144.000,00$ & $86 \%$ & $\mathrm{R} \$ 123.840,00$ & $\mathrm{R} \$ 1.593 .800,00$ \\
\hline 16 & $\mathrm{R} \$ 144.000,00$ & $85 \%$ & $\mathrm{R} \$ 122.400,00$ & $\mathrm{R} \$ 1.716 .200,00$ \\
\hline 17 & $\mathrm{R} \$ 144.000,00$ & $84 \%$ & $\mathrm{R} \$ 120.960,00$ & $\mathrm{R} \$ 1.837 .160,00$ \\
\hline 18 & $\mathrm{R} \$ 144.000,00$ & $83 \%$ & $\mathrm{R} \$ 119.520,00$ & $\mathrm{R} \$ 1.956 .680,00$ \\
\hline 19 & $\mathrm{R} \$ 144.000,00$ & $82 \%$ & $\mathrm{R} \$ 118.080,00$ & $\mathrm{R} \$ 2.074 .760,00$ \\
\hline 20 & $\mathrm{R} \$ 144.000,00$ & $81 \%$ & $\mathrm{R} \$ 116.640,00$ & $\mathrm{R} \$ 2.191 .400,00$ \\
\hline 21 & $\mathrm{R} \$ 144.000,00$ & $80 \%$ & $\mathrm{R} \$ 115.200,00$ & $\mathrm{R} \$ 2.306 .600,00$ \\
\hline 22 & $\mathrm{R} \$ 144.000,00$ & $79 \%$ & $\mathrm{R} \$ 113.760,00$ & $\mathrm{R} \$ 2.420 .360,00$ \\
\hline 23 & $\mathrm{R} \$ 144.000,00$ & $78 \%$ & $\mathrm{R} \$ 112.320,00$ & $\mathrm{R} \$ 2.532 .680,00$ \\
\hline 24 & $\mathrm{R} \$ 144.000,00$ & $77 \%$ & $\mathrm{R} \$ 110.880,00$ & $\mathrm{R} \$ 2.643 .560,00$ \\
\hline 25 & $\mathrm{R} \$ 144.000,00$ & $76 \%$ & $\mathrm{R} \$ 109.440,00$ & $\mathrm{R} \$ 2.753 .000,00$ \\
\hline
\end{tabular}

Fonte: Dados da Pesquisa (2020).

Os resultados mostram que em menos de 3 anos (sem considerar taxa de juros e inflação) a instituição pesquisada teria de volta em economia o valor que investiu e o longo de 25 anos economizaria $\mathrm{R} \$ 2.753 .00,00$.

Abaixo destaca-se o Valor Presente Líquido - VPL, para tanto foi utilizado a seguinte formula: 


$$
V_{P L}=\sum_{n=1}^{n=N} \frac{F c_{t}}{(1+i)^{n}}
$$

Onde:

VPL $=$ Valor Presente Líquido

$\mathrm{FC}=$ fluxo de caixa

$\mathrm{t}=$ momento em que o fluxo de caixa ocorreu

i = taxa de desconto (ou taxa mínima de atratividade)

$\mathrm{n}=$ período de tempo

Através do cálculo do Excel om uma Taxa Mínima de Atratividade de 12\%, o VPL= R \$902.503,73, ou seja, na análise da viabilidade desse projeto, mostra-se que descontando as taxas de juros obtém-se a verdadeira noção do valor do dinheiro no futuro (Assaf Neto, 2010).

No segundo cálculo foi avaliado a Taxa Interna de Retorno que é a taxa que irá igualar o fluxo de caixa antecipado para o valor do investimento. Para o cálculo foi utilizado a seguinte formula (LUNKES, 2011).

$$
0=V P L=\sum_{t=0}^{T} \frac{F C_{t}}{(1+T I R)^{t}}
$$

Onde:

VP L= Valor Presente Líquido;

Capital $=$ valor do investimento;

$\mathrm{T}=$ quantidade de períodos;

Fct $=$ entrada de capital no período t;

$\mathrm{TIR}=$ taxa interna de retorno.

Através do cálculo do Excel a Taxa Interna de Retorno correspondeu a 34\%, o que demostra que o investimento e viável. No terceiro cálculo foi realizado o Payback que é o tempo de retorno desde o investimento inicial até o momento em que os rendimentos acumulados se tornam iguais ao valor desse investimento. A tabela abaixo mostra a variação e o saldo em cada ano (Assaf Neto, 2010).

Tabela 5 - Payback descontado.

\begin{tabular}{ccccc}
\hline Ano & \multicolumn{2}{c}{ Fluxo } & Fluxo descontado & Saldo \\
\hline & 0 & $-\mathrm{R} \$ 415.000,00$ & $-\mathrm{R} \$ 415.000,00$ & $-\mathrm{R} \$ 415.000,00$ \\
& 1 & $\mathrm{R} \$ 144.000,00$ & $\mathrm{R} \$ 128.571,43$ & $-\mathrm{R} \$ 286.428,57$ \\
& $\mathrm{R} \$ 142.560,00$ & $\mathrm{R} \$ 113.647,96$ & $-\mathrm{R} \$ 172.780,61$ \\
& & $\mathrm{R} \$ 100.446,43$ & $-\mathrm{R} \$ 72.334,18$ \\
& $\mathrm{R} \$ 141.120,00$ & $\mathrm{R} \$ 88.769,17$ & $\mathrm{R} \$ 16.434,98$ \\
& $\mathrm{R} \$ 139.680,00$ & $\mathrm{R} \$ 78.441,09$ & $\mathrm{R} \$ 94.876,07$ \\
\hline
\end{tabular}

Fonte: Dados da Pesquisa (2020). 
Analisando a tabela, traves do cálculo do payback descontado o tempo de retorno desde o investimento inicial é de 3,8 anos. Nesse sentido o payback trata-se de uma estratégia, um indicador usado nas empresas para calcular o período de retorno de investimento em um projeto.

Por fim a tabela 6 mostra a decisão de investimento, ou seja, se a instituição tivesse pego esse capital R\$415.000,00 e investido em outras áreas no mercado financeiro.

Tabela 6 - Decisão de Investimento.

\begin{tabular}{clc}
\hline Investimento & Valor de resgate & Taxa líquida média \\
\hline Tesouro Selic & $\mathrm{R} \$ 3.025 .966,80$ & $8,27 \%$ \\
Poupança & $\mathrm{R} \$ 1.852 .962,47$ & $6,17 \%$ \\
Fundos DI & $\mathrm{R} \$ 2.338 .849,70$ & $7,16 \%$ \\
CDB & $\mathrm{R} \$ 2.838 .206,16$ & $7,99 \%$ \\
LCI e LCA & $\mathrm{R} \$ 2.630 .954,89$ & $7,67 \%$ \\
Energia Solar & $\mathrm{R} \$ 3.168 .000,00$ & - \\
\hline
\end{tabular}

Fonte: Valor Investe (2020).

A análise e decisão de possíveis investimentos consiste na utilização de técnicas contábeis e financeiras para determinar qual o investimento é mais rentável à organização. O uso dessa metodologia de decisão permite ao gestor identificar qual projeto possui o maior potencial de rentabilidade para os negócios, norteando os próximos passos da organização (Assaf Neto, 2010).

Dentre as opções de mercado a poupança aparece como o pior investimento. O tesouro Selic foi o que mais rendeu dentre as opções de mercado rendeu $\mathrm{R} \$$ 3.025.966,80, mesmo assim ficou ainda atrás da rentabilidade e da economia trazida pela Energia Solar ao longo de 25 anos com o valor de R \$3.168.000,00. Vale destacar que não foram considerados mudanças na taxa de juros e inflação.

\section{Considerações Finais}

Um dos enormes desafios no mundo hoje está relacionado a utilização dos diferentes tipos de energia de forma sustentável e eficiente. Compreender os cenários envolvidos na matriz energética no mundo pode ser um fator determinante para o futuro das gerações. E esse entendimento contribuiu para o desenvolvimento significativo de fontes renováveis, substituindo as tradicionais que agridem o meio ambiente.

Desse modo, a adoção de sistemas de Energia Solar pode trazer benefícios claros a população. O valor unitário de energia mostra que, de modo geral, a produzida pelo sistema é mais em conta que o praticado pela concessionária. Ou seja, o produtor está economizando toda vez que consumir a energia que seu próprio sistema a produz.

Apesar do custo de elevado implantação desse sistema, ele apresenta um curto prazo de retorno financeiro. Observa-se que o retorno do investimento é relativamente baixo se comparado com a garantia que os painéis fotovoltaicos possuem, que é cerca de 25 anos de geração, chegando ao vigésimo quinto ano com $76 \%$ de rendimento. Os módulos tem garantia de 10 anos contra defeitos e os inversores possuem 8 anos de garantia.

Os resultados mostraram que o prazo para o retorno de investimento está em 3,8 anos, como pode ser visto na Tabela 8. Ainda de acordo com os resultados espera-se que haja uma economia de cerca de $\mathrm{R} \$ 2.753 .000,00$ nos próximos 25 anos, o que torna a instalação do sistema bastante atrativa. Uma comparação entre diferentes tipos de investimento foi realizada, demonstrando o quão importante é a utilização dos painéis solares para a economia energética e financeira nos próximos anos. 
Para todas as propostas verificadas, os valores calculados para Payback, do Valor Presente Líquido e da Taxa Interna de Retorno mostram-se superiores aos prazos e taxas mínimas de atratividade. Pode-se concluir que a implantação deste sistema é economicamente viável, sendo que o seu tempo de retorno.

Sendo assim, é evidente o benefício que a utilização das fontes alternativas pode trazer tanto para as organizações, quanto ao meio ambiente, beneficiando a população como um todo. O Brasil tem um enorme potencial quanto o assunto, que é energia solar e com certeza pode ser mais bem explorada por todos segmentos da indústria brasileira e mundial.

Para trabalhos futuros recomenda-se uma profunda análise, com a necessidade de projeção futura acerca do crescimento da instituição, até uma estabilização da mesma quanto à estrutura física e quantidade de alunos, sendo possível assim, prever o dimensionamento e o investimento para que o sistema continue atendendo toda a demanda de energia da instituição. Por fim, é importante ressaltar a relevância do desenvolvimento de um trabalho como este, no sentido da disseminação desta tecnologia, bem como das fontes renováveis de energia em geral.

\section{Referências}

Alves, D. R. da S., \& Lira, M. A. T. (2018). Estudo de viabilidade técnica e econômica para implantação de energia solar fotovoltaica em unidade consumidora no aeroporto de Teresina no Estado do Piauí. In: VII Congresso Brasileiro de Energia Solar.

Alves, G., Moraes, L., Marafão, F., Serni, P., \& Simões, M. (2014). Energia solar no Brasil. O Setor Elétrico, São Paulo, ed. 104.

ANEEL. (2020). Nota Técnica $n^{\circ} 0129 / 2012$.

Assaf Neto, A. (2010). Finanças corporativas e valor. 5. ed. São Paulo: Atlas.

Brasil. (2012). Resolução $n^{\circ} 482$, de 17 de 2012. Dispõe da instalação de sistemas de microgeração e minigeração distribuídas conectadas à rede de distribuição. ANEEL.

Cabral, I. de S., \& Vieira, R. (2012). Viabilidade econômica x viabilidade ambiental do uso de energia fotovoltaica no caso brasileiro: uma abordagem no período recente. In: III Congresso Brasileiro de Gestão Ambiental.

Climate-Data.Org. (2020). Clima Cajazeiras - PB: Climedata.org, 2020. https://pt.climate-data.org/america-do-sul/brasil/paraiba/cajazeiras-42515/.

Cruz, R. A. da. (2020). Estudo de viabilidade técnica e econômica para geração de eletricidade através do uso de células fotovoltaicas. Trabalho de Conclusão de Curso: apresentado ao curso MBA em Gestão Empresarial, de Pós-Graduação lato sensu, Nível de Especialização, da FGV/IDE.

Dantas, S. G., \& Pompermayer, F. M. (2018). Viabilidade econômica de sistemas fotovoltaicos no Brasil e possíveis efeitos no setor elétrico. Texto para discussão/Instituto de Pesquisa Econômica Aplicada. Brasília: Rio de Janeiro: Ipea.

EPE. (2017). Empresa de Pesquisa Energética. Anuário Estatístico de Energia Elétrica 2017. Ano base. Rio de Janeiro: EPE.

Instituto Brasileiro de Geografia e Estatística. (2012). Censo 2012: IBGE. http://www.ibge.gov.br/home/.

Ishii, T., \& Masuda, A. (2017). Annual degradation rates of recent crystalline silicon photovoltaic modules. Progress In Photovoltaics: Research and Applications, (1)25, 953-967.

Google Earth. (2020). Mapa via satélite: Cidade de Cajazeiras - PB. Google. https://www.google.com.br/intl/pt-BR/earth/.

Krauss, K., Fertig, F., Menzel, D., \& Rein, S. (2015). Light-induced degradation of silicon solar cells with aluminium oxide passivated rear side. Energy Procedia, (2) $77,599-606$.

Lunkes, R. J. (2011). Manual do orçamento. 2. ed. 5. reimpr. São Paulo: Atlas.

Maia, R. S. (2018) Energia solar: o desenvolvimento de um novo mercado. Tese de Doutorado: Universidade Federal do Rio de Janeiro.

Marchioro, A. F., Dahmer, R. D., \& Souza, R. C. (2018). Metodologia para análise da viabilidade técnica-econômica para geração de energia fotovoltaica. In: VII Congresso Brasileiro de Energia Solar: Gramado.

Malhotra, N. (2014). Pesquisa de marketing. 5.ed. Porto Alegre: Bookman

Marconi, M. A., \& Lakatos, E. M. (2012). Técnicas de Pesquisa. São Paulo: Atlas.

Mori, I. Y. (2018). Análise da viabilidade de um sistema de geração distribuída solar fotovoltaica para UTFPR Londrina. Trabalho de Conclusão de Curso: apresentado como requisito parcial para obtenção do Título de Bacharel em Engenharia Ambiental da Universidade Tecnológica Federal do Paraná, Câmpus Londrina.

Oliveira, S. L. (2012). Tratado de metodologia científica. São Paulo: Pioneira Thomson. 
Research, Society and Development, v. 10, n. 6, e19510615645, 2021 (CC BY 4.0) | ISSN 2525-3409 | DOI: http://dx.doi.org/10.33448/rsd-v10i6.15645

Schossler, M. H. (2018). Estudo de viabilidade financeira para implantação de sistemas de energia solar fotovoltaica em residências e empreendimentos comerciais do município de Lajeado. Trabalho de Conclusão de Curso: Engenharia Civil, da Universidade do Vale do Taquari: Univates.

Siqueira, L. M. de. (2015). Estudo do Dimensionamento e da Viabilidade Econômica de Microgerador Solar Fotovoltaico Conectado à Rede Elétrica. Trabalho de Conclusão de Curso: apresentado a Faculdade de Engenharia da Universidade Federal de Juiz de Fora.

Sonai, G. G., Melo, J. R. M. A., Nunes, J. H. B., Megiatto, J. R. J. D., \& Nogueira, A. F. (2015). Células solares sensibilizadas por corantes naturais: um experimento introdutório sobre energia renovável para alunos de graduação. Quim. Nova, (10)38, 1357-1365.

Valor Investe. (2020). Calculadora de taxas: Investimento. https://valorinveste.globo.com/ferramentas/calculadoras/investimentos/. 